\title{
Presymptomatic testing for Huntington's disease in the United Kingdom
}

\author{
Audrey Tyler, David Ball, David Craufurd on behalf of the United Kingdom Huntington's Disease \\ Prediction Consortium
}

Abstract

Objective-To evaluate the United Kingdom Huntington's disease presymptomatic testing programme.

Design-Postal questionnaire survey to collect data on all tests performed by clinical genetics centres between 1987 and 1990 .

Setting-Genetic centres providing presymptomatic testing in the United Kingdom.

Subjects -248 subjects at risk of Huntington's disease who had presymptomatic testing at their request.

Main outcome measures-Sex, age, prior risk, and risk after testing.

Results-The risk of carrying the Huntington disease gene was reduced for $151(61 \%)$ of the applicants and raised for $97(39 \%) .158(64 \%)$ of the subjects were female and $90(36 \%)$ male. The median age at which the results were given was 32.5 years.

Conclusions-The demand for testing was lower than expected and may have reached its peak in 1990. The excess of low risk results was not fully explained by the age effect. All the genetics centres concerned have agreed a common service protocol which requires extensive pre-test counselling and post-test follow up. The worth of the procedure remains to be decided. The availability of a large body of pooled data from all the United Kingdom testing centres, which individually are likely to have only a few results, will form a valuable resource for monitoring the long term psychosocial impact of testing.

Institute of Medical Genetics, University of Wales College of Medicine, Cardiff CF4 4XW

Audrey Tyler, medical social worker (research)

David Ball, research registrar

St Mary's Hospital, Manchester M13 0JH David Craufurd, senior lecturer in genetics

\section{United Kingdom}

Huntington's Disease

Prediction Consortium Genetics centres that are members of the consortium are listed at the end of this report.

Correspondence to: $\mathrm{Ms}$ Tyler.

BMF 1992;304:1593-6

\section{Introduction}

Huntington's disease is a progressive neurological disorder inherited as an autosomal dominant trait. The clinical features typically appear during the fourth and fifth decades of life with restlessness and involuntary movements which develop into severe chorea and dystonia with generalised motor impairment as the disease progresses. Affected subjects are prone to a wide range of psychiatric disorders together with a subcortical dementia often causing profound difficulties in the regulation of behaviour. Death occurs usually about $15-20$ years after onset.

Since 1983 the availability of genetic markers closely linked to the gene for Huntington's disease has permitted presymptomatic testing for those at risk. ${ }^{1} 3^{2}$ These markers are not specific to Huntington's disease but are present in the general population, and testing is therefore possible only when the pattern of the markers co-inherited with the gene in a particular family can be distinguished from those markers inherited from the unaffected side of that family. For this to be achieved DNA samples from both parents and another affected family member or a grandparent are normally the minimum requirement. As Hunting- ton's disease is an autosomal dominant disorder a person at risk has an equal chance of inheriting an affected or unaffected chromosome from the affected parent. If identification of the affected chromosome is possible the person's prior risk of $50 \%$ can be substantially raised or lowered ${ }^{3}$ (fig 1).

Presymptomatic testing offers obvious advantages for those given a low risk, but there are very serious implications for those given an unfavourable result, perhaps many years before symptoms are destined to appear. Potential ethical difficulties associated with such tests include the possibility of adverse psychological effects on both the subject and the family as well as the likelihood of discrimination in such matters as employment and insurance. ${ }^{45}$ Recognition of these problems prompted the drawing up of internationally agreed guideline ${ }^{67}$ and a growing appreciation of the need for a system to monitor and regulate the tests being carried out. This led to the establishment in 1990 of the United Kingdom Huntington's Disease Prediction Consortium, and all the centres currently offering presymptomatic testing in Britain have agreed to participate. The aims of the collaborative group are (a) to promote and maintain good standards of service provision, $(b)$ to agree a common protocol for this purpose, $(c)$ to serve as a forum for debate, and $(d)$ to collect data to evaluate the testing programme.

We present a survey of clinical genetic centres that are members of the consortium and an analysis of the data collected, covering the first four years of predictive testing.

\section{Methods}

The first presymptomatic tests for Huntington's disease in the United Kingdom were performed in 1987. Over the next three years the number of centres offering testing rose from two to 19 and 16 centres gave out test results. After the inception of the collaborative group data were collected by questionnaire from these centres for the period up to 1 January 1991. For each complete test performed within a given year the sex, age, prior risk, and risk after testing of the subject were requested. From our comprehensive knowledge of the centres offering testing in the United Kingdom we regard the information presented as virtually complete.

As the gene for Huntington's disease has not been identified the test results have a margin of error due to the possibility of genetic recombination between the markers and the gene. This occurs during meiosis, when chromosomes can "cross over"; the further away the markers are from the gene the greater is the possibility of recombination. The original marker, G8, has been calculated to have a recombination rate of $4 \% .^{1}$ Newer markers now in use for predictive testing are closer to the gene and their recombination rate has been calculated to be between $1 \%$ and $2 \% .^{2}$ 
Calculating post-test risk therefore depends on three factors: $(a)$ the closeness of the markers which determine informativeness; $(b)$ the number of meioses which distance the applicant from the relatives used to determine informativeness-if DNA samples from grandparents are not available, which is usually the case, other relatives such as affected uncles and aunts or siblings are frequently used, and in these circumstances the margin of error will be greater; $(c)$ the age of the applicant at the time of testing-the older the applicant the lower the likelihood of an individual having the Huntington disease gene.

In view of the complexities it is essential that the final risk given is calculated by using a computerised program, such as MLINK. ${ }^{8}$

\section{Results}

Between 1987 and 1990, 248 presymptomatic tests were performed in the United Kingdom - two in 1987, 42 in 1988, 108 in 1989, and 96 in 1990 . The number of tests completed varied widely from centre to centre. Six regional genetic centres were responsible for 192 results in almost equal proportions, the remaining 56 results (range 1-16) having been shared among 10 centres.

One hundred and fifty eight $(64 \%)$ subjects tested were female and $90(36 \%)$ male. The ages at which subjects were given results are presented in the table. The median age overall was $32 \cdot 5$ years (range 18-62). The median ages at which female and male subjects received results were 32 and 33 years respectively. Only seven subjects, all female, were under 20 . More than a quarter of the results were given to subjects between the ages of 25 and 29 years, and $189(76 \%)$ subjects were under 40 .

Five subjects had a prior risk of $25 \%$ - that is, they had an affected grandparent and the intervening parent was not known to be clinically affected. In these circumstances the risk can be altered to only a limited extent. Two subjects had a prior risk of $36 \%$ as their parents' risk had been altered to $72 \%$ by previous testing. The average age and percentage of those receiving high and low risk results were not altered when this small subgroup was removed; therefore, the data are not analysed separately. The remaining subjects (241) were at $50 \%$ prior risk. The age corrected risks before testing are presented in figure 2 . A high risk result (range 70-98\%) was given to 97 (39\%) subjects; a low risk result (range $1-25 \%$ ) was given to $151(61 \%)$ subjects, and in over 100 of these the risk could be lowered to less than $4 \%$. There was no significant difference in the proportions of men and women who received high and low risk results.

\section{Discussion}

During 1987 to 1989 there was an increase in the number of presymptomatic tests performed annually within the United Kingdom, but the figure for 1990 indicated a moderate fall, which, on an individual centre basis, seemed to relate to the year testing began. Centres which started testing in 1987 and 1988 showed a downward trend in numbers in 1990; centres which started later were showing an upward trend. The peak

Age and sex distribution of subjects having presymptomatic testing for Huntington's disease during 1987-90

\begin{tabular}{lcccccccccccc}
\hline & \multicolumn{10}{c}{ Age (in five year categories) } \\
\cline { 2 - 13 } & $<20$ & $20-$ & $25-$ & $30-$ & $35-$ & $40-$ & $45-$ & $50-$ & $55-$ & $60-64$ \\
\hline No of men & 0 & 12 & 22 & 14 & 17 & 8 & 6 & 6 & 3 & 2 \\
No of women & 7 & 17 & 44 & 27 & 29 & 19 & 4 & 8 & 2 & 1 \\
\hline Total & 7 & 29 & 66 & 41 & 46 & 27 & 10 & 14 & 5 & 3 \\
\hline
\end{tabular}

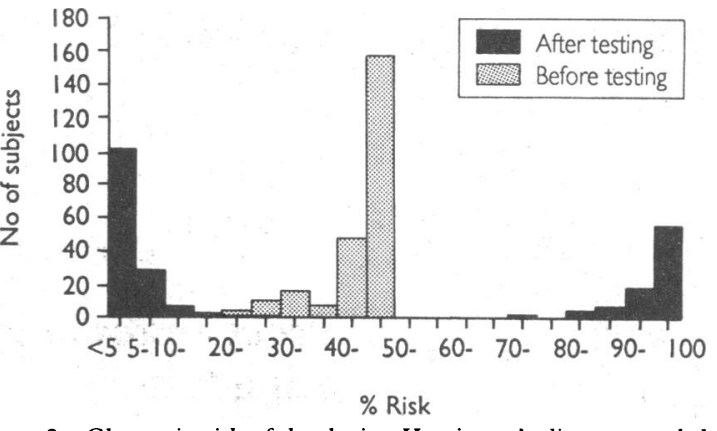

FIG 2-Change in risk of developing Huntington's disease recorded after testing

of activity in 1989 probably reflected the clearing of a build up of people who had been waiting for some time for the introduction of the test. The 1990 figure may therefore give a better estimate of demand for tests in the future. The downward trend may continue until the isolation of the gene permits testing from an individual sample of DNA, when an increase in demand is again anticipated.

Demand for testing has been much less than expected as surveys carried out before linkage analysis was possible suggested that between $56 \%$ and $66 \%$ of people at risk would make use of a predictive test if it were available..$^{10}$ The total of 248 tests performed up to 1 January 1991 represented a small proportion of subjects at $50 \%$ risk for the disease, estimated at around 10000 based on population prevalence estimates." Almost two thirds of those choosing presymptomatic testing were female, a finding consistent with reports from elsewhere. ${ }^{12-14}$ It has been suggested that this difference reflects greater maternal involvement in reproductive decision making or concern for existing children at risk, or both..$^{12-14}$ It is also possible that men may experience greater difficulty in accepting the implications of being at risk and are less able or willing to deal with the emotions that risk alteration arouses, coping by denial rather than confronting the issue..$^{15}$ The absence of teenage males (in the testing series) supports the clinical observation that this group is the least likely to accept genetic counselling.

That more people received a lowered than a raised risk (151 compared with 97) may be due in part to their age distribution. As age increases there is a reduction in the chance of developing Huntington's disease because some cases will already have presented. The effect may be exaggerated because normal neurological findings are generally a requirement for entry to the predictive testing programme and any subjects showing equivocal signs are therefore likely to have been excluded. However, the median age of people having presymptomatic testing was 32 years, and the age adjusted risk for a subject with a $50 \%$ prior risk at this age calculated by life table analysis would have fallen only to $46 \%$. $^{7}$ The expected bias towards low risk results would therefore be $54 \%$ to $46 \%$, so it seems unlikely that age alone can account for the bias towards favourable test results.

There may also be a process of self selection operating in people seeking presymptomatic testing. Given the low uptake of testing, quite possibly those who seek it may be unrepresentative of the at risk population as a whole. This may occur because some at risk subjects correctly perceive that they are free of subtle abnormalities becoming evident in their siblings and are thus encouraged to seek predictive testing in the expectation of a favourable result. Alternatively, it may be the result of personality factors and cognitive changes associated with the prodromal stages of the disease which reduce the motivation to be tested. Denial of the diagnosis and of the presence of symptoms 
is common in affected subjects during the early stages of Huntington's disease, and it is a frequent clinical observation that at risk subjects who have maintained a high level of vigilance for symptoms throughout their lives become oblivious to these when disease onset occurs. Some presymptomatic carriers of the Huntington disease gene may therefore be less likely to seek predictive testing because early cognitive changes have made them less worried about the possibility of inheriting the disease. Conversely, others may have become aware of subtle prodromal symptoms that cannot be detected clinically and avoid testing in order to delay confrontation of this fact.

CORE PROTOCOL FOR SERVICE USE

Because of the complexity of the procedures, the need for strict diagnostic accuracy, and concern about the psychological and social consequences for those given an unfavourable prediction, one of the first tasks of the consortium was to produce a universally accepted service protocol. This states that applicants should have a confirmed family history of Huntington's disease, have a potentially informative family structure, be aged 18 years and over, and freely give informed consent. The decision whether to be tested is always the choice of the individual, except for limited exclusion criteria which include $(a)$ people already clinically affected with Huntington's disease, $(b)$ people having a serious suicidal intent, $(c)$ people suffering from current mental illness, and $(d)$ people for whom there is reason to believe that their mental health could be significantly impaired by testing.

Preparation for the test consists of at least two counselling sessions separated by a significant interval and conducted by two counsellors, one of whom should be medically qualified and the other an experienced genetic counsellor. Applicants should also have previously received full genetic counselling in an appropriate centre. When there is a stable relationship the partner will always be asked to participate in at least one counselling session.

At the first counselling session basic demographic information is collected and the methods and limitations of testing explained, including the need for blood samples from family members and confirmation of the diagnosis in key relatives. Applicants' knowledge of the disease, the impact of being at risk, and their motivation for taking the test are explored. The potential consequences of receiving a result are discussed, with particular reference to possible adverse effects on the applicant's mental health; marital, family, and social relationships; employment; financial and insurance commitments; and plans. Subjects are also encouraged to consider in advance to whom the test result will be disclosed and who will provide posttest support. Written material is offered to reinforce the counselling and a neurological examination is performed at either the first or second interview.

Applicants presenting with neurological signs can be a difficult counselling problem because they are often unaware or unwilling to admit that they could already be affected ${ }^{16}$ If the signs of the disease are unmistakable the tester usually suggests that the test should be postponed and a formal neurological opinion obtained when the patient is ready. An equally difficult situation can arise when the symptoms are equivocal; the experienced tester may be highly suspicious but the symptoms are insufficient to permit confirmation of the diagnosis. If the patient remains firm in the desire to be tested, after careful observation, assessment, and counselling molecular genetic testing may be performed, but this procedure can be hazardous. These patients in effect suffer a double blow: they discover not only that they are gene carriers but that they are already showing signs of the disease, and one case of serious self harm has already been reported in such a circumstance in Canada. ${ }^{17}$

The second counselling session takes place when it is known that testing the applicant would be informative. At that session the post-test medical and social supports available are identified, a psychological evaluation is completed, and the disclosure of the test result to any third parties is reviewed. The arrangements for travel to the "disclosure" session and the disclosure session itself are rehearsed. Applicants are strongly recommended to bring a companion with them and not to drive themselves. Finally, applicants sign a consent form giving permission for their DNA to be typed.

Post-test counselling is always offered. Four sessions are scheduled in the first year and annually thereafter, at which further psychological evaluation is carried out. However, the extent to which people tested want and use post-test counselling is variable.

\section{TIMING OF TESTING}

Two groups of applicants need special consideration: teenagers and people newly at risk. Although maturity is not necessarily a function of chronological age, it is advisable to take special care with teenagers and allow them time for reflection as they may have particular problems in comprehending the possible long term consequences of an unfavourable result.

Likewise it is advisable to allow considerable time (usually a year) to elapse between making the diagnosis of Huntington's disease in the parent and accepting any of their offspring into the testing programme. Experience shows that people newly at $50 \%$ risk initially express great interest in having their risk refined but change their minds later when they have adjusted to their new status and have given more thought to the possible impact of an adverse result.

\section{Conclusion}

The worth of presymptomatic testing in Huntington's disease remains to be proved and requires careful follow up of subjects tested. The availability of a large body of pooled data from all the presymptomatic testing centres in the United Kingdom will form a valuable resource for monitoring the long term impact of these tests on the patients and families concerned.

We acknowledge the contribution made by all the members of the consortium, and particularly the following: Alan Johnston and Sheila Simpson (Aberdeen); Patrick Morrison and Norman Nevin (Belfast); Sarah Bundey and Ian Glass (Birmingham); Peter Lunt and Valerie Reader (Bristol); German Berrios, Clare Davison, Eamonn Maher, and John Yates (Cambridge); Peter Harper and Michael Morris (Cardiff); David Brock and Alan Wright (Edinburgh); Heather May and John Tolmie (Glasgow); Martin d'a Crawfurd, Elizabeth Thompson, and Robin Winter (Harrow); Gwen Turner (Leeds); Judith Anderson and Jayne Shakespeare (Leicester); Alan Fryer (Liverpool); Shirley Dalby (Huntington's Disease Association, London); Anita Harding (Institute of Neurology, London); Ann Howie (S George's Hospital, London); Shirley Hodgson and Alison Lashwood (Guy's Hospital, London); Michael Patton (St George's Hospital, London); Rodney Harris and Lauren Kerzin-Storrar (Manchester); William Barker, John Burn, and Dorothy Gibson (Newcastle); Sandy Raeburn (Nottingham); Anona Galliard, Susan Huson, and Richard Lindenbaum (Oxford); Nick Dennis, Christine Patch, and Christopher Ward (Southampton); Oliver Quarrell (Sheffield); and Heather Skirton (Taunton).

Clinical genetics centres that are consortium members and to which referrals may be made are: Aberdeen Royal Infirmary, Aberdeen AB9 2YS (0224 681818); Belfast City Hospital Tower, Belfast, Northern Ireland BT9 7AB (0232 229241); Birmingham Maternity Hospital, Birmingham B15 2TG (021 472 1377); Bristol Children's Hospital, Bristol BS2 8BJ (0272 215411); Addenbrooke's Hospital, Cambridge CB2 2QQ (0223 345151); University Hospital of Wales, Cardiff CF4 
4XN (0222 747747); Western General Hospital, Edinburgh EA4 2HU (031 3327917); Duncan Guthrie Institute, Glasgow G3 8SJ (041 339 8888); Kennedy Galton Centre, Northwick Park Hospital, Middlesex HAl 3UJ (081 422 8577); Clarendon Wing, General Hospital, Leeds LS2 9NS (0532 432799); Leicester Royal Infirmary, Leicester LE1 7LX (0533 541414); Royal Liverpool Hospital, Liverpool L69 3BX (051 709 0141); Institute of Child Health, London WCIN 1EA (071 242 7989); Seventh and Eighth Floors, Guy's Tower, Guy's Hospital, London SE1 9RT (071 955 4648); St George's Hospital Medical School, London SW 17 ORE (071 672 9944); St Mary's Hospital, Manchester M13 0JH (061 276 6322); 19/20 Claremont Place, Newcastle upon Tyne NE2 4AA (0912 226000); City Hospital, Nottingham NG5 1PB (0602 691169); Churchill Hospital, Oxford OX3 7LE (0865 62834); 117 Manchester Road, Sheffield S10 5DN (0742 27511); Princess Anne Hospital, Southampton SO9 4HA (0703 796166).

1 Gusella JF, Wexler NS, Conneally PM, Naylor SL, Anderson MA, Tanzi RE et al. A polymorphic DNA marker genetically linked to Huntington's et al. A polymorphic DNA mark

2 Hayden MR, Robbins C, Allard D, Haines J, Fox S, Wasmuth J, et al. Improved predictive testing for Huntington's disease by using three linked DNA markers. Am $\mathcal{Y}$ Hum Genet 1988;43:689-94.

3 Harper PS, ed. Predictive tests in Huntington's disease. In: Huntington's disease. Major problems in neurology. Vol 22. London: W B Saunders, 1991:373-413

4 Craufurd D, Harris R. Ethics of predictive testing for Huntington's chorea: the need for more information. BMJ 1986;293:249-51.
5 Morris M, Tyler A, Harper PS. Adoption and genetic prediction for Huntington's disease. Lancet 1988;ii:1069-70.

6 World Federation of Neurology: Research Committee Research Group on Huntington's Chorea. Ethical issues policy statement on Huntington's disease molecular genetics predictive test. F Neurol Sci 1989;94:327-32.

Harper PS, Newcombe RG. Age at onset and life table risks in genetic counselling for Huntington's disease. F Med Genet 1992;29:239-43.

8 Sandkuyl L, Ott J. Determining informativity of marker typing for genetic counselling in a pedigree. Hum Genet 1989:82:159-62.

Tyler A. Harper PS. Antudes of subjects at risk and their relatives towards (1983;20:179-88. genetic counselling in Huntington's chorea. $\mathcal{F}$ Med Genet $1983 ; 20: 179-88$.
Mastromauro C, Myers RH, Berkman B. Attitude towards presymptomatic testing in Huntington's disease. Am F Hum Genet 1987;26:271-82.

11 Harper PS, ed. The epidemiology of Huntington's disease. In: Huntington's Harper PS, ed. The epidemiology of Huntington's disease. In: Huntington's
disease. Major problems in neurology. Vol 22. London: W B Saunders, 1991:251-80

12 Craufurd D, Dodge A, Kerzin-Storrar L, Harris R. Uptake of presymptomatic predictive testing for Huntington's disease. Lancet 1989;ii:603-5.

13 Meissen GA, Myer RH, Mastromauro C, Koroshetz WJ, Klinger KW, Farrer LA, et al. Predictive testing for Huntington's disease with use of a linked DNA marker. N Engl f Med 1988;318:535-42.

14 Brandt J, Quaid KA, Folstein SE, Garber P, Maestri NE, Abbott MH, et al. Presymptomatic diagnosis of delayed-onset disease with linked DNA markers. The experience in Huntington's disease. FAMA 1989;261:3108-14.

15 Bloch M, Fahy M, Fox S, Hayden MR. Predictive testing for Huntington's disease; demographic characteristics, lifestyle patterns, atritudes and psydisease, demographic characteristics, lifestyle pattens, attitudes and psy1989;32:217-24.

16 Tyler A, Morris M, Lazarou L, Meredith L, Myring J, Harper PS, et al Presymptomatic testing for Huntington's disease in Wales 1987. 1990. Br $\mathcal{J}$ Psychiatry (in press)

17 Lam RW, Bloch M, Jones BD, Marcus AM, Fox S, Amman W, et al. Psychiatric morbidity associated with early clinical diagnosis of Huntington disease in a predictive testing programme. F Clin Psychiatry 1988;49:444-7.

(Accepted 23 March 1992)

\title{
Electrocardiographic abnormalities and associated factors in Chinese living in Beijing and in Mauritius
}

Beijing Heart Lung and Blood Vessel Medical Centre, Beijing, China Nan Li, research fellow Zhang Min, systems analyst You Chonghua, director

\section{Department of} Epidemiology, National Public Health Institute 00510 Helsinki, Finland Jaakko Tuomilehto, professor

International Diabetes Institute, Caulfield, Australia

Gary Dowse, epidemiologist Paul Zimmet, professor

Medical School, Newcastle upon Tyne

K George Alberti, professor

Noncommunicable Diseases Office, Ministry of Health, Port Louis, Mauritius

Pierrot Chitson, medical officer

Hassam Gareeboo, medical officer

World Health Organisation, Port Louis, Mauritius

Sir Djamil Fareed, WHO representative

Correspondence to: Professor J Tuomilehto, Department of Epidemiology, National Public Health Institute, Elimäenkatu 25A 6th Floor 00510 Helsinki, Finland.

BMF 1992;304:1596-601

\author{
Nan Li, Jaakko Tuomilehto, Gary Dowse, K George Alberti, Paul Zimmet, Zhang Min, Pierrot \\ Chitson, Hassam Gareeboo, You Chonghua, Djamil Fareed for the Mauritius Non-Communicable \\ Disease Study Group
}

Abstract
Objective-To compare the prevalence of electrocardiographic abnormalities and to evaluate the association between these abnormalities and the levels of coronary heart disease among Chinese living in different environments.

Design-Cross sectional surveys.

Setting - Beijing, China, and the island of Mauritius.

Subjects-Random samples of people aged 35-64 years in Beijing (621 men, 642 women) in 1984 and in Mauritius among Chinese ( 137 men, 130 women) and non-Chinese (1265 men, 1432 women) in 1987.

Main outcome measures-Prevalence of electrocardiographic abnormalities suggesting coronary heart disease and of associated risk factors.

Results-Prevalence of electrocardiographic abnormalities suggesting coronary heart disease was significantly lower in Beijing (4.0\%) than in Mauritian Chinese (24.3\%) and Mauritian non-Chinese (24.5\%). Mean serum concentrations of total and non-high density lipoprotein cholesterol were lower in Beijing Chinese than in Mauritian Chinese, but smoking and hypertension were slightly more prevalent. Overall, men with electrocardiographic abnormalities had higher risk factor levels than those with a normal electrocardiogram regardless of ethnic origin.

Conclusions-The prevalence of coronary heart disease and associated risk factors was different among Chinese living in two different environments: in Beijing in the People's Republic of China and in Mauritius. Chinese, who traditionally have a very low frequency of coronary heart disease, are by no means protected against coronary heart disease and other non-communicable diseases. Therefore, primary prevention of coronary heart disease is a major challenge for preventive medicine in China, as well as in many other developing countries.

\section{Introduction}

International comparisons have shown that the occurrence of coronary heart disease varies greatly from one country to another. In China the incidence of and mortality from coronary heart disease are low, as shown by, for example, the World Health Organisation monitoring trends and determinants in cardiovascular disease (MONICA) project. ${ }^{1}$ The highest age standardised coronary heart disease mortality in men aged 35-64 years was found in the Siberian MONICA study $(401 / 100000)$ and the lowest in the SinoMONICA-Beijing study (40/100000). In women the highest coronary heart disease mortality was found in the Glasgow MONICA study (132/100000) whereas in Beijing it was only $28 / 100000 .^{1}$ These 10 -fold differences in men and $4 \cdot 7$-fold differences in women may be explained by different levels of coronary heart disease risk factors, ${ }^{2}$ but it has also been proposed that these risk factors may have different impact on the development of coronary heart disease in different geographic areas and in genetically different populations.

There have been many studies comparing coronary heart disease risk factor levels in different countries. ${ }^{2-6}$ Genetics, ethnic background, lifestyles, and study methods have often differed between the areas, hence the possibilities for valid conclusions from many earlier comparisons are limited. The Ni-Hon-San (NipponHonolulu-San Francisco) Japanese migrant study initiated in the mid-1960s overcame many methodological shortcomings and showed a clear gradient in coronary heart disease mortality among Japanese men 